Gefässchirurgie 2010 $15: 511-512$ DOI 10.1007/s00772-010-0860-4

Online publiziert: 29. Oktober 2010

๑) Springer-Verlag 2010

\author{
H.-H. Eckstein \\ Klinik für Gefäßchirurgie, Klinikum rechts der Isar (MRI) \\ der Technischen Universität München
}

\title{
Weiterbildung in der Gefäßchirurgie
}

Liebe Kolleginnen, liebe Kollegen,

Nach Angaben der Bundesärztekammer wurden im vergangenen Jahr 25 Kolleginnen und 64 Kollegen als Fachärzte/innen für Gefäßchirurgie anerkannt. Hinzu kommen weitere Schwerpunktsbezeichnungen nach alter WBO. Leider stehen diese Zahlen auf Bundesebene nicht exakt zur Verfügung. Insgesamt kann jedoch von 100-120 neuen Gefäßchirurgen/-innen im Jahr 2009 ausgegangen werden. Gleichzeitig waren zum Stichtag 31.12.2009 insgesamt 1.206 Gefäßchirurgen/-innen berufstätig. Diese Zahl ist im Vergleich zum 31.12.2008 erneut um $6,4 \%$ bzw. $n=73$ gestiegen. Der etwas geringere Anstieg der Gesamtzahl berufstätiger Gefäßchirurgen/-innen gegenüber den neuen Gefäßchirurgen/-innen ist darauf zurückzuführen, dass eine Reihe von Kollegen/-innen die Altersgrenze erreicht hat oder aus anderen Gründen aus dem aktiven Job ausgestiegen ist.

Die versorgungsmedizinisch wichtigste Frage ist natürlich, ob diese neuen Gefäßchirurgen/-innen den Bedarf decken, eine Frage, die derzeit klar mit Nein beantwortet werden muss. Ein Blick auf den sog. Facharztindex, der regelmäßig im Deutschen Ärzteblatt (DÄB) publiziert wird, verdeutlicht die Situation. Für den Facharztindex wird die Zahl der für ein Fachgebiet im DÄB veröffentlichten Stellenanzeigen ins Verhältnis zur Zahl der in diesem Fachgebiet angestellt tätigen Ärztinnen und Ärzte gesetzt. Auf diese Weise erhält man einen spezifischen Indexwert, der angibt, wie viele Fachärztinnen und Fachärzte rein rechnerisch auf eine Stellenausschreibung entfallen. Je niedriger der Indexwert ausfällt, desto geringer ist für Fachärztinnen und Fachärzte die Zahl potenzieller Mitbewerber. Bei einem Indexwert unter 10, wird von einer sehr dünnen Bewerberdecke ausgegangen.

(2) Gefäßchirurgen/-innen gehören seit Jahren zu den am meisten gesuchten Fachärzten

Die - Tab. 1 zeigt eindrucksvoll, dass sich die Gefäßchirurgie seit Jahren regelmäßig unter den am meisten gesuchten Facharztdisziplinen überhaupt befindet. Wenn man weiterhin berücksichtigt, dass nur ein kleinerer Teil aller Stellen überhaupt noch ausgeschrieben wird (jede Klinik ist heilfroh, wenn frei werdende Stellen aus dem eigenen Nachwuchs besetzen werden können), wird das eigentliche Ausmaß des Nachwuchsmangels an-

\begin{tabular}{|lllll|}
\hline Tab. $\mathbf{1}$ Facharztindex & $\mathbf{2 0 0 6}$ & $\mathbf{2 0 0 7}$ & $\mathbf{2 0 0 8}$ & $\mathbf{2 0 0 9}$ \\
Facharzttitel & 8,4 & 8,3 & 5,8 & 6,6 \\
Gefäßchirurgie & 8,1 & 6,5 & 8,8 & 6,7 \\
Viszeralchirurgie & 8,9 & 5,4 & 6,1 & 6,4 \\
\hline Gastroenterologie & 5,3 & 7,5 & 6,1 & 7,9 \\
\hline Kinder- und Jugendpsychiatrie & 8,7 & 8,4 & 9,4 & 6,9 \\
Hämatologie/Onkologie & 7,2 & k.A. & 9 & 7,8 \\
Thoraxchirurgie & 17,9 & 14,6 & 14,1 & 16 \\
\hline Durchschnittswert aller Fachgebiete & & & &
\end{tabular}




\section{In eigener Sache}

satzweise deutlich. Allein 2008 wurden 133 Oberarztstellen für Gefäßchirurgie im DÄB ausgeschrieben, mithin kann jede/r frische Facharzt/-ärztin davon ausgehen, umgehend eine Oberarztposition erhalten zu können. Ob dies für manche Kollegen nicht doch etwas früh kommt, bleibt dahingestellt, die seit Jahren angespannte Lage diktiert dieses Vorgehen.

Insgesamt ist überdeutlich, dass die Gefäßchirurgie boomt (entgehen aller Schwarzmalerei). Ebenso klar ist aber auch, dass eine quantitativ und qualitativ hochwertige Weiterbildung zwingend notwendig ist. Deshalb geht es darum, die Attraktivität unseres Fachs darzustellen und gleichzeitig ein inhaltlich anspruchsvolles Curriculum zu gewährleisten. Die Einladung von mehr als 40 Medizinstudenten $\mathrm{zu}$ unserer letzten Jahrestagung war diesbezüglich eine absolut wertvolle Initiative des Kongresspräsidenten Sebastian Debus. Der nächste Schritt wird nun die Möglichkeit einer studentischen Mitgliedschaft in der DGG sein.

Auch im Namen der Schriftleitung bedanke ich mich bei allen Autoren, die sich in diesem und dem nächsten Heft der Gefässchirurgie mit verschiedenen Aspekten der gefäßchirurgischen Weiterbildung auseinandersetzen. Ich hoffe sehr, dass es uns gemeinsam gelingt, das Thema Weiterbildung als eine fortwährende Aufgabe und einen ständigen Prozess zu begreifen, um die unverkennbaren Fortschritte der Gefäßmedizin künftig noch schneller in unser Fach integrieren zu können.

Mit den besten Grüßen Ihr

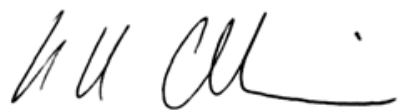

Prof. Dr. H.-H. Eckstein

\section{Korrespondenzadresse Prof. Dr. H.-H. Eckstein}

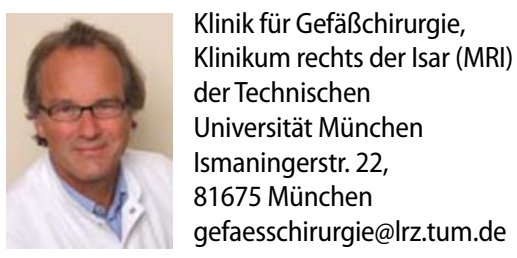

\section{www.SpringerMedizin.de Das Internet der Ärzte}

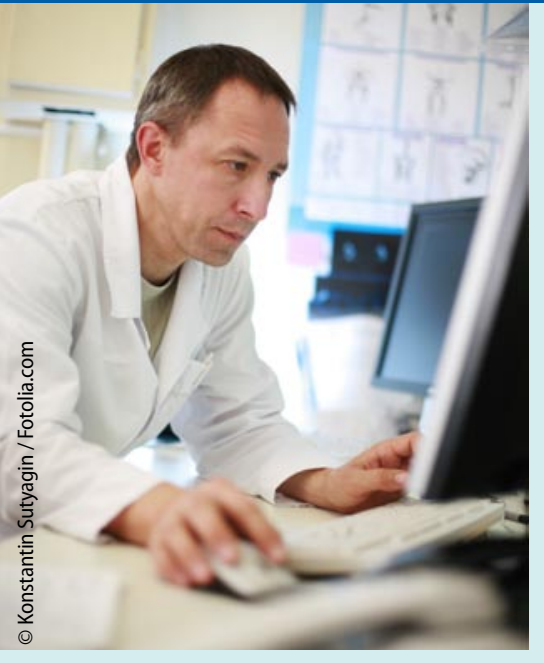

Sie möchten auf dem Laufenden bleiben? In umfassenden Zeitschriftenbibliotheken recherchieren? Sich fortbilden und CME-Punkte sammeln oder bei Experten Ihres Fachgebiets Rat suchen? Dann sind Sie auf SpringerMedizin.de, dem neuen Fachportal für Ärzte, goldrichtig. Denn dort erwartet Sie eine Fülle von nützlichen Informationen - maßgeschneidert auf Ihr Fachgebiet.
Titeln geordnet. In dieser umfassenden Online-Bibliothek stehen für Abonnenten die Inhalte von über 70 renommierten Titeln bis in die 1990er-Jahre bereit.

Ihr personalisiertes Informationsangebot

SpringerMedizin.de ist ein Fachportal für Ärzte, daher sind alle medizinischen Inhalte passwortgeschützt. Die kostenlose Registrierung ist schnell erledigt und hat einen großen Vorteil: Sobald Sie bei der Registrierung Ihr Fachgebiet angegeben haben, erhalten Sie künftig automatisch als personalisierte Startseite das entsprechende Fachportal angezeigt. Ihre Startseite können Sie nach Wunsch weiter anpassen: Die grauen Rubrikenkästen (sog. Portlets) lassen sich verschieben, umgruppieren oder auch wegklicken. Über den Knopf „Mehr Inhalte hinzufügen" können Sie weitere Rubriken auswählen und auf der Startseite anzeigen lassen. Möchten Sie auch News und Beiträge aus anderen Fachgebieten lesen - kein Problem, konfigurieren Sie Ihr Portlet ganz

\section{Von Anaesthesie bis Zahnmedizin - die ganze Welt der Medizin ist}

nur wenige Klicks entfernt. www.SpringerMedizin.de ist das Fachportal für Ärzte in Deutschland

Es lohnt sich, mehrmals am Tag mit SpringerMedizin.de online zu gehen. Täglich stellt Ihnen das Redaktionsteam neue interessante Themen aus Medizin und Gesundheitspolitik zusammen. Weitere News aus der Hauptstadt und Informationen, was sich in den einzelnen Bundesländern und KVen tut, stehen im Rubrikenkasten „Gesundheitspolitik" bereit. Täglich neue Meldungen zu medizinischen Themen finden Sie unter "Medizin kompakt". Kurz und bündig geben Ihnen diese Meldungen einen schnellen Überblick, was sich in der Medizin alles tut.

\section{Fortbildung und $\mathrm{CME}$}

Unter dem Navigationspunkt „Fortbildung“ stehen Ihnen interessante Übersichtsarbeiten, spannende Kasuistiken und CMEBeiträge aus den Springer-Fachzeitschriften zur Verfügung. Unter „Zeitschriften“ finden Sie das gesamte Zeitschriftenangebot von Springer Medizin nach Fachgebieten und nach Wunsch. Sobald Sie die Konfiguration abgespeichert haben, erscheint sie beim nächsten Log-in genau so wieder. Über den orangenen Button „Guided Tour" erklären wir Ihnen alle Möglichkeiten der individuellen Anpassung in einem Film.

Diese Beispiele sind nur ein Bruchteil dessen, was SpringerMedizin.de zu bieten hat. Steuern Sie das Internet der Ärzte an und lassen Sie sich überraschen! Es lohnt sich.

\section{Dr. Sonja Kempinski \\ Chefredakteurin SpringerMedizin.de}

